\title{
Alpha-synuclein Fibrils Structure Determination in the Presence of Tau Using CryoEM
}

Alimohammad Hojjatian ${ }^{1}$, Anvesh Dasari ${ }^{2}$, Dianne Taylor ${ }^{1}$, Nadia Daneshparvar ${ }^{1}$, Fatemeh Abbasi Yeganeh ${ }^{1}$, Kwang $\lim ^{2}$ and Kenneth Taylor ${ }^{1}$

${ }^{1}$ Florida State University, Tallahassee, Florida, United States, ${ }^{2}$ East Carolina University, Greenville, North Carolina, United States

With the resolution revolution in cryo-EM structure determination [1], near atomic resolution of 3Dreconstruction became almost routinely possible opening the way for a "future which is not crystallized". Due to the low Signal to Noise Ratio of $\sim 3 \%$ in electron micrographs of frozen hydrated biological samples [2], a vast number of particles must be aligned iteratively, starting from low resolution frequencies [3], in order to produce a near atomic resolution $2 \mathrm{D}$ or $3 \mathrm{D}$ reconstruction while avoiding overfitting or incorrect fitting [4]. The presence of symmetry can be of enormous significance, as it can basically limit the alignment to a portion of the molecule with the rest replicated using symmetry. Helical symmetry, for example, a Helix can normally be described with three parameters: helical rise, helical twist and handedness of the helix [5-7]. Helical symmetry was of enormous help in solving the first high resolution structure of a biological sample by cryoEM using Fourier-Bessel inversion [5]. However, every high-level symmetry comes with great risk of amplification of noise in search of signal that can result in overfitting or wrong structure determination. $\alpha$-synuclein fibrils, as amyloids, are of great pathological significance in so-called synucleinopathies but their structural features are very difficult to reconstruct in 3D. Amyloid fibril structure is generally described as long pitch helix of densely stacked ( $4.75 \AA$ spacing) flat subunits (subunit sheets are oriented perpendicular to the helical axis) in an assumed left handed helix. Subunit packing within the filament is also known as cross-beta-sheet quaternary structure which leaves one direction of structure without any structural features except for the $4.75 \AA$ axial spacing between adjacent subunits [8]. Implementation of iterative helical real-space reconstruction algorithm in RELION [10] enabled us to reconstruct and correct for imperfect helices instead of using Fourier-Bessel inversion which could not be applied to imperfect helices [9], as many studies show helical cross-over distance variations from one filament to the next [8]. To avoid possible local optima, we checked different values for helical parameters and after all of the refinements our structure resolution went down to $4.0 \AA$ with almost 200,000 segments, starting from roughly 1,000,000 segments. Using MonoRes [11] and LocalDeblur [12] gave us better sharpening of the map. Due to the relatively low resolution, we were not able to build the atomic model from de novo, as well the handedness determination of filaments was not possible for resolutions lower than 2.3 $\AA$. However, our ssNMR data showed that the resonances for certain residues in our filaments are as those of a previously reported structure (PDB: 6rt0) for $\alpha$-synuclein filaments. Using this as an initial model, we used Coot and added alanine residues, mutated them with the correct amino acids and refined the atomic model. Continuing efforts involve removing or lowering the clashes between a couple of the residues and improving the Ramachandran scores which are not satisfactory. Side chains are not generally fully resolved which makes the process more complicated. A tomography data set was collected from which the filaments were shown to be left handed. Our study is differentiated from other studies by the presence of Tau monomers with the $\alpha$-synuclein. Tau is a pathologically related amyloid has implications in synucleinopathies. Previous studies shown that tau interacts with $\alpha$-synuclein and accelerate $\alpha$-synuclein aggregation. In this study, we investigated the effect of tau on $\alpha$-synuclein aggregates and found that our $\alpha$-synuclein filaments have distinct protofilament interactions than those of previously reported $\alpha$-synuclein filaments. Despite 2D results that show extra densities on the outer surface of the $\alpha$-synuclein filaments, we were not able to resolve them in $3 \mathrm{D}$ reconstructions. However, the 
tomography data set combined with subvolume averaging may resolve the extra densities (projected to be Tau proteins) in 3D without the need to impose helical symmetry. The refinement of the atomic model, validation and subvolume averaging is still in progress. The Study is funded by NIH.
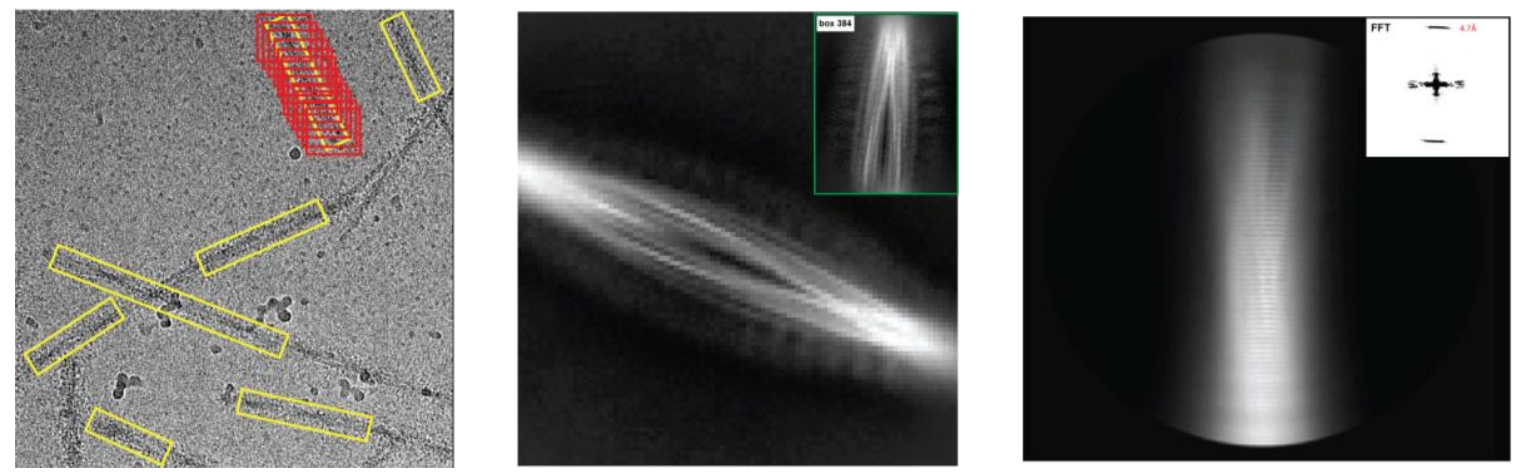

Figure 1. Left: shows an electron micrograph, tubes are shown by yellow boxes and red boxes show the procedure of helical extraction subsequent to the manual helical picking in RELION. Middle: non-helical 2D alignment and classification using cisTEM (box $844 \AA$ ) and green box shows the same procedure using a smaller box size that shows the outer surface extra densities more clearly. Right: shows a class average and the Fourier transform of the same class after helical 2D alignment and classification.
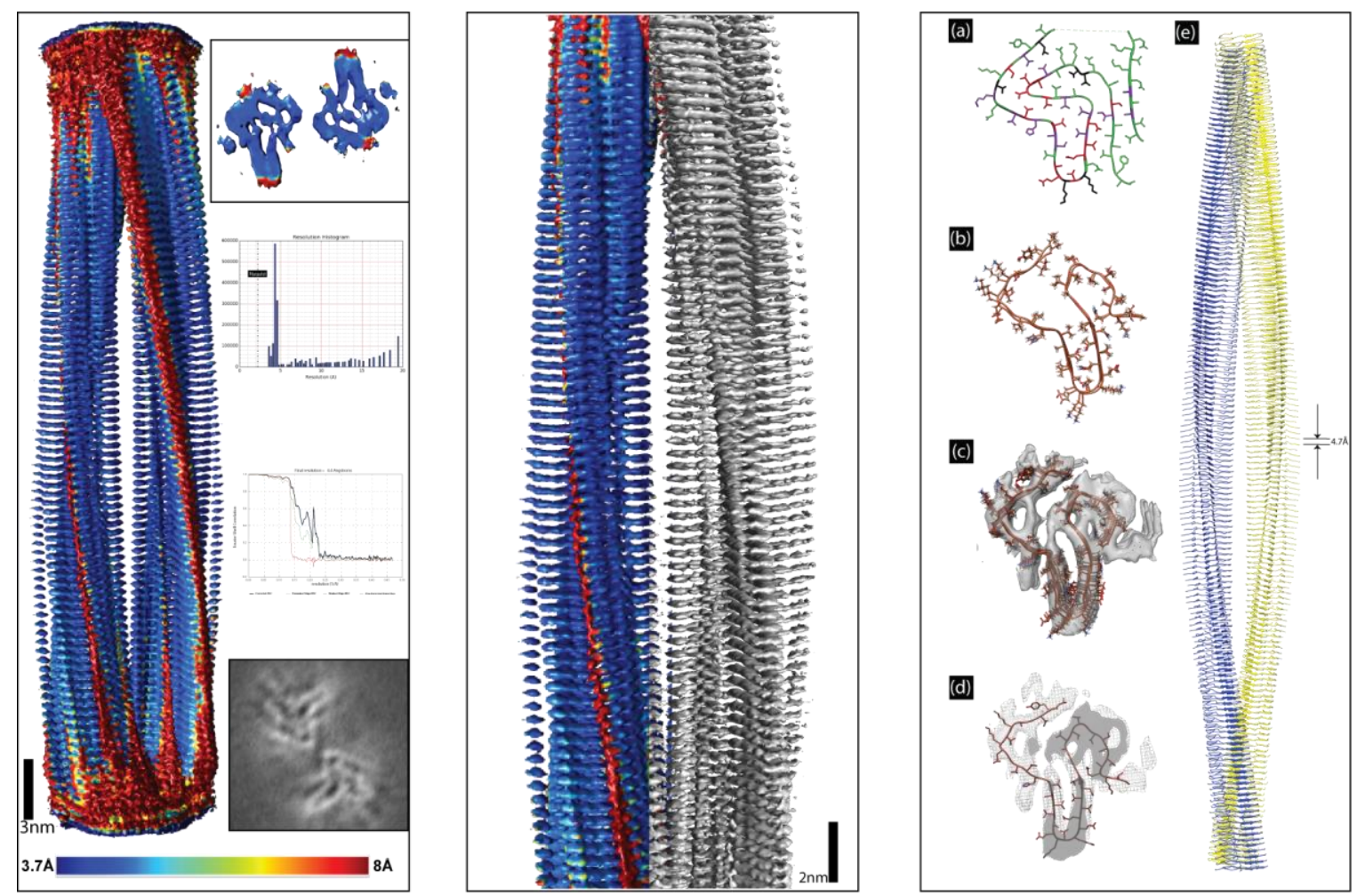

Figure 2. Left: shows the result of local resolution determination using MonoRes and FSC. Middle: shows the locally sharpened map (grey right half) using LocalDeblur compared to the result of globally sharpened (colorful left half) using RELION. Right: (a) shows the result of NMR study on the similarity of the resonance on 6rt0. (b-e) show the atomic model refined in Coot, Rosetta and Phenix. 


\section{References}

1. Callaway, E. (2015). The revolution will not be crystallized: a new method sweeps through structural biology. Nature News, 525(7568), 172.

2. Frank, J., \& Al-Ali, L. (1975). Signal-to-noise ratio of electron micrographs obtained by cross correlation. Nature, 256(5516), 376-379.

3. Frank, J. (2006). Three-dimensional electron microscopy of macromolecular assemblies: visualization of biological molecules in their native state (New York: Oxford University Press)

4. Henderson, R. (2013). Avoiding the pitfalls of single particle cryo-electron microscopy: Einstein from noise. Proceedings of the National Academy of Sciences, 110(45), 18037-18041.

5. Cochran, W., Crick, F. H., \& Vand, V. (1952). The structure of synthetic polypeptides. I. The transform of atoms on a helix. Acta Crystallographica, 5(5), 581-586.

6. Klug, A., Crick, F. H. C., \& Wyckoff, H. W. (1958). Diffraction by helical structures. Acta Crystallographica, 11(3), 199-213.

7. He, S., \& Scheres, S. H. (2017). Helical reconstruction in RELION. Journal of structural biology, 198(3), 163-176.

8. Scheres, S. H. (2020). Amyloid structure determination in RELION-3.1. Acta Crystallographica Section D: Structural Biology, 76(2).

9. Egelman, E. H. (2000). A robust algorithm for the reconstruction of helical filaments using singleparticle methods. Ultramicroscopy, 85(4), 225-234.

10. Zivanov, J., Nakane, T., Forsberg, B. O., Kimanius, D., Hagen, W. J., Lindahl, E., \& Scheres, S. H. (2018). New tools for automated high-resolution cryo-EM structure determination in RELION-3. Elife, 7 , e42166.

11. Vilas, J. L., Gómez-Blanco, J., Conesa, P., Melero, R., de la Rosa-Trevín, J. M., Otón, J., ... \& Sorzano, C. O. S. (2018). MonoRes: automatic and accurate estimation of local resolution for electron microscopy maps. Structure, 26(2), 337-344.

12. Ramírez-Aportela, E., Vilas, J. L., Glukhova, A., Melero, R., Conesa, P., Martínez, M., ... \& Marabini, R. (2020). Automatic local resolution-based sharpening of cryo-EM maps. Bioinformatics, 36(3), 765772 . 